\title{
Patrones y peculiaridades del apego romántico en adultos de 62 regiones culturales: ¿Son los Modelos de Sí Mismo y Otros constructos panculturales?
}

David Schmitt*

Martín Nader**

Maria Martina Casullo***

\section{Abstract}

As a part of the International Sexuality Description Project, a total of 17,804 participants from 62 cultural regions completed the Relationship Questionnaire (RQ), a self-report measure of adult romantic attachment. Correlational analyses within each culture suggested that the "Model of Self" and "Model of Other" scales of the RQ were psychometrically valid within the most cultures. Contrary to expectations, the Model of Self and Model of Other dimensions of the RQ did not underlie the four category model of attachment in the same way across all cultures. Analyses of specific attachment styles revealed that Secure romantic attachment was normative in $79 \%$ of cultures, and Preoccupied romantic attachment was particularly prevalent in East Asian cultures. Finally, the romantic attachment profiles of individual nations were correlated with sociocultural indicators in ways that supported evolutionary theories of romantic attachment and basic human mating strategies.

Key Words: Model of Self. Model of Other.

\section{Resumen}

Como parte del proyecto internacional de descripción de la sexualidad, un total de 17804 participantes de 62 regiones culturales completaron el Relationship Questionnaire, un autoinforme que mide el apego romántico en adultos. Diversos análisis correlacionales hechos dentro de cada cultura sugieren que las escalas modelo de sí mismo y modelo de otro del RQ son psicométrica-

\footnotetext{
* Bradley University. Illinois. U.S.A. Coordinador general del ISDP.

** Secretaria de Ciencia y Tecnología de la Nación. Argentina. Becario.

*** CONICET. UBA. Coordinadora responsable en Argentina.
} 
mente validas en la mayoría de las culturas estudiadas. Contrario a lo esperado, las dimensiones modelo de sí mismo y modelo de otro del RQ no integran el modelo de cuatro categorías de apego de la misma manera en todas las culturas. Distintos análisis específicos sobre estilos de apego mostraron que el apego seguro normativo está presente en el $79 \%$ de las culturas, mientras que el apego romántico preocupado es especialmente prevalente en culturas del sudeste asiático. Finalmente, los perfiles de apego de las distintas naciones están correlacionados con indicadores socioculturales de manera que confirman empíricamente teorías evolutivas sobre apego romántico y estrategias humanas básicas de elección de pareja.

Palabras Clave: Modelo de Sí Mismo. Modelo del Otro. 


\section{Introducción}

En este articulo se presentan los resultados hallados en el Proyecto Internacional para la Descripción de la Sexualidad (ISDP). El ISDP fue desarrollado con el propósito de obtener evaluaciones psicológicas acerca de la sexualidad de una extensa variedad de culturas. Como parte del proyecto, más de 17000 participantes de 62 regiones culturales distintas completaron un autoinforme denominado Relationship Questionnaire (Bartholomew y Horowitz, 1991). El mismo mide apego romántico en adultos.

Se plantearon cinco objetivos que guiaron la investigación. El primero de ellos fue determinar si las escalas modelo de sí mismo y modelo de otro del RQ eran válidas en todas las culturas estudiadas en el ISDP. El segundo objetivo fue evaluar si esas dos dimensiones subyacían a los cuatro estilos de apego romántico - seguro, despreocupado, preocupado y temeroso (ver Bartholomew, 1990) de la misma manera en todas las culturas estudiadas por el ISDP. El tercer objetivo fue determinar si la forma segura de apego es normativa en todas las culturas (van Ijzedoorn y Sagi, 1999). El cuarto objetivo fue investigar si los asiáticos del este son particularmente propensos al estilo de apego preocupado (Soon y Malley-Morrison, 2000). El quinto y último objetivo fue poner a prueba varias teorías evolutivas sobre apego romántico y estrategias humanas básicas de elección de pareja (Belsky, Steinberg y Draper, 1991; Chisholm, 1996). Debido a que la investigación sobre apego romántico en adultos estuvo muy influenciada por teorías evolutivas sobre apego (ver Cassidy y Shaver, 1999; Simpson y Rholes 1998), presentamos una breve revisión de los orígenes evolutivos del apego.

\section{Orígenes evolutivos del apego}

Siguiendo la teoría etológica del apego de Bowlby $(1969,1982)$, los seres humanos poseemos un sistema motivacional-conductual que surge en la infancia y está diseñado para proteger a los niños mientras pasan por distintas fases de su desarrollo (Marvin \& Brittner, 1999). Este sistema de apego es compartido por otros primates (Suomi, 1995) y ha evolucionado como un mecanismo adaptativo para monitorear la proximidad física y la disponibilidad de figuras protectoras de apego (Brethertorn \& Munholland, 1999). Un concepto central de esta teoría es que se supone que todos los niños deben pasar por las mismas fases de desarrollo y deben poseer los mismos sistemas de apego altamente evolucionados (van Ijseendorn \& Sagi, 1999). Generalmente se asume que el pasaje exitoso por todas las etapas universales del apego les brinda a los niños una base emocional segura, base que les permitirá conducirse en sus vidas con relaciones vinculares eficientes (Bowlby, 1988; Hazan \& Zeifman, 1999). 
Las experiencias tempranas de apego proveen a los chicos de una base emocional segura principalmente impactando sobre los sentimientos fundamentales de seguridad, mérito y confianza interpersonal (Bowlby, 1973, 1980). Las experiencias infantiles que incluyen cuidados, apoyo e interés por parte del cuidador producen un alto nivel de autoconfianza y un sentimiento duradero de confort a la hora de depender de otros. Estos pensamientos y sentimientos pueden cristalizar en modelos internos de trabajo o en actitudes emocionales-cognitivas que aseguran que el si mismo es valorable y merecedor de amor (p.ej., los chicos desarrollan un modelo positivo de si mismo) y que los otros son valorables y merecedores de amor (p.ej, los chicos desarrollan un modelo positivos de otros). Las experiencias de cuidados irresponsables, de abuso o inconsistentes, en contraste, generan modelos internos de trabajo negativos o disfuncionales.

Los modelos disfuncionales pueden consistir de un modelo negativo de los otros (por desconfianza y baja valoración de los padres), un modelo de si mismo negativo (baja autoestima y sensibilidad al rechazo) o modelos negativos tanto del si mismo como de los otros (Bartholomew, 1990). Eventualmente, estos modelos internos de trabajo pueden convertirse en componentes estables del núcleo de personalidad del chico: "una vez construido, según los hallazgos, estos modelos de padres y del si mismo interactuantes tienden a persistir, se dan por sentados y empiezan a operar a nivel inconsciente" (Bowlby, 1988, p. 130).

\section{Modelos internos de trabajo y apego romántico adulto}

A mediados de los '80, se comenzó a investigar de que manera los estilos de apego podían influir en las actitudes cognitivo emocionales hacia el amor romántico y las relaciones sexuales (Hazan y Shaver, 1987). Desde la última década, un número creciente de evidencia ha mostrado que las diferentes orientaciones influyen profundamente en las maneras en las que las personas piensan y sienten las relaciones románticas. Las variaciones en el apego adulto han sido atribuidas a patrones conflictivos de relaciones románticas y stress (Rholes, Simpson y Stevens, 1998; Simpson, 1990), armonía y satisfacción romántica (Brennan y Shaver, 1995; Collins y Read, 1990; Roberts \& Noller, 1998), así como a la duración temporal de las relaciones románticas (Hazan y Zeifman, 1999; Kirkpatrick, 1998; Simpson, 1999). En general, las personas con un estilo de apego seguro tienden a experimentar menos conflicto, mayor satisfacción, gran estabilidad y una mayor duración de sus relaciones románticas (Belsky, 1999; Kirkpatrick y Hazan, 1994).

Siguiendo el modelo de dos factores de apego romantico de Bartholomew y Horowitz (1991), los modelos internos de trabajo son actualmente dimensiones independientes que, en combinación, forman cuatro estilos básicos de apego romántico adulto (ver también Bartholomew, 1990). Las diferentes combinaciones de los mode- 
los de si mismo y de otros producen cuatro actitudes diferenciadas hacia las relaciones románticas. Por ejemplo, las personas que tienen modelos positivos de si mismo y otros presentan un estilo de apego romántico "seguro". Las personas seguras tienen sentimientos de que son dignos de captar la atención y el amor de otros. También poseen expectativas de que las otras personas aceptaran y reaccionaran a las expresiones de amor brindadas. Basado en el modelo de Bartholomew y Horowitz, estos sentimientos y pensamientos están generados por actitudes cognitivo emocionales inconscientes representadas internamente como modelos positivos de si mismo y otros.

Las personas despreocupadas también mantienen un modelo de si mismo positivo pero con un modelo negativo de otros. Esta combinación conduce a las personas a experimentar alta autoestima, pero tienden a autoprotegerse de desencuentros amorosos evitando relaciones mas intimas y manteniendo un sentido de independencia e invulnerabilidad. Los individuos preocupados muestran un modelo negativo de si mismos y un modelo positivo de otros. Este tipo de personas se esfuerzan continuamente por ser aceptados por otras personas, altamente valoradas por ellos. Los individuos miedosos o inseguros tienen modelos negativos de si mismo y de otros. Tienden a evitar relaciones románticas porque se ven a si mismos como no merecedores de recibir amor, ven el amor de los otros como no disponible por largo tiempo y esperan ser rechazados por sus parejas (Bartholomew \& Horowitz, 1991).

Las dos dimensiones básicas (modelo de cuatro categorías) presentado por Bartholomew \& Horowitz (1991) puede integrarse con otros modelos de apego romántico (Bartholomew \& Shaver, 1998; Griffin \& Bartholomew, 1994 b) y ha recibido gran apoyo proveniente de la literatura referente al apego (Brennan et al, 1998; Carver, 1997; Feeney, 1999). Sin embargo, la validación inicial del modelo se realizo con muestras relativamente pequeñas $(\mathrm{N}=77, \mathrm{~N}=69)$ y los estudios subsiguientes estuvieron limitados a las culturas occidentales (Bartholomew, 1994; Bartholomew \& Shaver, 1998; Scharfe \& Bartholomew, 1994).

Aun cuando muchos investigadores del apego consideraron a los procesos del desarrollo del apego - que dan lugar a los modelos internos de trabajo de si mismo y otros - como universales (Main, 1990; van Ijzendoorn \& Sagi, 1999), muchos de ellos argumentaron que los núcleos centrales de la teoría están muy sesgados por los estilos de pensamiento occidentales. Por ejemplo, Rothbaum y sus colegas recientemente cuestionaron si la base segura del apego promueve universalmente la adaptación a través de la exploración y la individuación (Rothbaum, Weisz, Pott, Miyake \& Morelli, 2000). Efectivamente, muchas de las diferencias culturales han estado implicadas como moderadores de las conductas de apego (Ainsworth \& Marvin, 1995). Debido a que el modelo mencionado no ha sido suficientemente estudiado en culturas no occidentales (Summer \& Gungor, 1999), permanece poco claro si es una característica universal de la psicología humana o si difiere considerablemente en diferentes culturas. 


\section{Lógica del presente estudio}

Explora la psicología del apego romántico adulto en 62 regiones culturales para responder a cinco objetivos primarios:

1. Determinar si las escalas Modelo de Si Mismo y Modelo de Otros son validas en todas las culturas estudiadas por el ISDP.

2. Determinar si las dimensiones Modelo de Si Mismo y Modelo de Otros subyacen de maneras similares a varios estilos de apego de diferentes culturas.

3. Determinar si la forma segura de apego romántico es normativa a través de todo el ISDP.

4. Determinar si los asiáticos del este puntúan particularmente alto en el estilo preocupado de apego romántico.

5. Determinar si los individuos de culturas con grandes recursos, pocos estresares sociales y bajas tasas de fertilidad poseen orientaciones de apego mas seguro.

Proveer información básica y descriptiva del apego romántico adulto a través de todas las culturas del ISDP puede resultar muy útil para su uso en futuras investigaciones. Los datos de los estilos de apego prevalentes en las diferentes culturas en un determinado momento resuelven muchos de los problemas que vienen surgiendo desde las década pasada en referencia a los estudios transculturales comparativos (Bartholomew \& Shaver, 1998). Si los patrones y universales de apego romántico existen a través de todas las culturas, el mejor método para detectarlas será un estudio longitudinal, como el realizado en el ISDP, en el que se recolecten datos de diferentes culturas con instrumentos que midan efectivamente apego romántico. Los resultados obtenidos en este proyecto contribuirán decisivamente al conocimiento de las naciones acerca de la socialización humana, su psicología y a la cultura en general.

\section{Método}

\section{Muestras}

El estudio presentado en este articulo es producto de ISDP (Proyecto Internacional para la Descripción de la Sexualidad), un esfuerzo de colaboración que resulto en una muestra de 17804 sujetos ( 7432 hombres y 10372 mujeres) de 62 regiones culturales de todo el mundo. Se presentan los resultados obtenidos en las muestras así como los métodos utilizados para la evaluación. 
Patrones y peculiaridades del apego romántico en adultos de 62 regiones culturales: ¿Son los Modelos de Sí Mismo y Otros constructos panculturales?

\begin{tabular}{|c|c|c|c|}
\hline Regiones Culturales & Lenguaje & $\begin{array}{c}\text { Hombres } \\
\mathrm{N}=\end{array}$ & $\begin{array}{c}\text { Mujeres } \\
\mathbf{N}=\end{array}$ \\
\hline \multicolumn{4}{|l|}{ NORTEAMERICA } \\
\hline Canadá inglés & Inglés & 313 & 553 \\
\hline Canadá francés & Francés & 60 & 113 \\
\hline USA Noroeste & Inglés & 72 & 156 \\
\hline USA Medio Oeste & Inglés & 184 & 357 \\
\hline USA Sur & Inglés & 368 & 570 \\
\hline USA Oeste & Inglés & 287 & 487 \\
\hline USA Hawai & Inglés & 88 & 224 \\
\hline México & Español & 106 & 109 \\
\hline \multicolumn{4}{|l|}{ SUDAMERICA } \\
\hline Perú & Español & 106 & 100 \\
\hline Bolivia & Español & 92 & 89 \\
\hline Chile & Español & 100 & 212 \\
\hline Argentina & Español & 110 & 136 \\
\hline Brasil & Portugés & 42 & 55 \\
\hline \multicolumn{4}{|l|}{ EUROPA DEL OESTE } \\
\hline Finlandia & Finlandés & 24 & 90 \\
\hline Norte Irlanda & Inglés & 56 & 244 \\
\hline Inglaterra & Inglés & 82 & 101 \\
\hline Holanda & Holandés & 115 & 126 \\
\hline Bélgica & Holandés/Flamenco & 166 & 356 \\
\hline Francia & Francés & 55 & 56 \\
\hline Suiza & Alemán & 85 & 129 \\
\hline Alemania & Alemán & 294 & 496 \\
\hline Austria & Alemán & 207 & 260 \\
\hline \multicolumn{4}{|l|}{ EUROPA DEL ESTE } \\
\hline Estonia & Estonio & 79 & 109 \\
\hline Letonia & Leton & 90 & 103 \\
\hline Lituania & Lituano & 47 & 47 \\
\hline Polonia & Polaco & 309 & 537 \\
\hline Rep. Checa & Checo & 106 & 129 \\
\hline Eslovaquia & Eslovaco & 84 & 100 \\
\hline Ucrania & Ucraniano & 100 & 100 \\
\hline Rumania & Rumano & 123 & 128 \\
\hline Serbia & Serbio & 100 & 100 \\
\hline Croacia & Croata & 113 & 109 \\
\hline Eslovenia & Eslovaco & 73 & 109 \\
\hline \multicolumn{4}{|l|}{ EUROPA DEL SUR } \\
\hline Portugal & Portugués & 110 & 142 \\
\hline España & Español & 95 & 178 \\
\hline Italia & Italiano & 92 & 108 \\
\hline Malta & Inglés & 133 & 198 \\
\hline Grecia & Griego & 47 & 182 \\
\hline Chipre & Griego & 24 & 36 \\
\hline \multicolumn{4}{|l|}{ MEDIO ORIENTE } \\
\hline Turquía & Turco & 206 & 206 \\
\hline El Líbano & Inglés & 124 & 139 \\
\hline Israel & Hebreo & 180 & 214 \\
\hline Jordania & Arabe & 80 & 195 \\
\hline
\end{tabular}


Esta colección de regiones culturales representa una muestra de las diversas etnias, religiones, y categorías lingüísticas. En total, las 62 regiones del ISDP representan a 6 continentes, 13 islas, 30 lenguajes y 56 naciones. Para algunas culturas (p.ej., Australia, Austria, Canadá, Chile, Inglaterra, Alemania, Israel, Malta, Corea del Sur, Turquía y las regiones Medio Oeste, Sur y Oeste de Estados Unidos) fue necesario recolectar más de una muestra. En otras, estuvieron involucrados varios colaboradores.

Muchas de las muestras están conformadas por estudiantes universitarios. En todos los casos, las muestras fueron intencionales. Muchos de los evaluadores fueron voluntarios, otros recibieron créditos universitarios y otros recibieron una pequeña remuneración económica por el trabajo. En todas las muestras, se administro una encuesta anónima auto administrable. Algunas de ellas volvieron en cartas selladas y otras fueron depositadas en los buzones de los respectivos departamentos. El porcentaje de encuestas devueltas por los estudiantes (como parte de su entrenamiento) es de $95 \%$.

\section{Procedimiento}

Todos los colaboradores del ISDP fueron instruidos para administrar una encuesta de 9 páginas a por lo menos 100 hombres y 100 mujeres. Como se observa en la tabla 1, muchos colaboradores se aproximaron bastante a las cifras planteadas. Los participantes recibieron un breve instructivo acerca del motivo de este estudio incluyendo el anonimato de las encuestas que contestarían, que varió de acuerdo a cada colaborador según la cultura en la que se encontrase. La encuesta se toma en aproximadamente 20 minutos.

\section{Instrumentos}

\section{Procedimiento para traducción}

Los investigadores de las diferentes regiones culturales que no tuvieran el ingles como lengua oficial fueron instruidos para aplicar un procedimiento de traducción-retraducción para administrar la encuesta en el idioma propio de esa cultura. Este proceso involucraba a un investigador que traducía la encuesta al idioma propio de su cultura (p.ej., castellano) y luego un segundo investigador retraducía la encuesta al inglés. Como se constata en la tabla 1, la encuesta fue traducida a más de 30 lenguas distintas. 


\section{Evaluación del apego romántico adulto}

A todas las muestras se les administro un instrumento de dos dimensiones y cuatro categorías denominado Relationship Questionnaire (Bartholomew \& Horowitz, 1991). Esta técnica tiene un ítem que mide apego seguro: "Es fácil para mi volverme emocionalmente cercano a otros. Me siento cómodo dependiendo de otros y otros dependiendo de mí. No me preocupa estar solo o sentir que otros no me aceptan". Los participantes disponen de una escala de tipo Likert para evaluar el ítem seguro. Tiene un rango que va desde 1 a 7 en donde 1 es "no me describe" y 7 "me describe de forma precisa" con 4 como punto medio. Puntajes altos en este ítem indican que el sujeto posee modelos internos de trabajo positivos de Si Mismo y Otros.

El RQ tiene tres ítems más que miden estilos de apego inseguro. El primero de ellos es el tipo de apego evitativo: "Me siento cómodo sin relaciones emocionalmente cercanas. Es muy importante para mi sentirme independiente y autosuficiente y prefiero no depender de otros o que otros dependan de mi". Puntajes altos indican un modelo de Si Mismo positivo y un modelo de Otros negativo. Un puntaje alto en el ítem del estilo de apego preocupado: "Quiero estar emocionalmente cercano con otros pero encuentro que los otros son reacios a estar conmigo de la manera que yo quiero. Me siento incomodo sin una relación emocionalmente intima pero a veces me preocupa que los otros no me valoren tanto como yo los valoro a ellos" indica un modelo negativo de Si Mismo y un modelo Positivo de Otros. El ultimo de los estilos de apego, el miedoso, dice: "Me siento incomodo si me acerco emocionalmente a otros. Quiero tener relaciones intimas pero encuentro difícil confiar en otros lo mismo que depender de otros o que otros dependan de mi. Me preocupa salir lastimado si me acerco emocionalmente a otros". Un puntaje alto en este ítem indica una persona con modelos de $\mathrm{Si}$ Mismo y otros negativos.

\section{Evaluación de la autoestima}

Todos los participantes completaron la Escala de Autoestima de Rosenberg (Rosenberg, 1965). Esta escala contiene cuatro ítems, cada uno de los cuales es evaluado por el sujeto en una escala formato Likert donde las evaluaciones van desde Totalmente de acuerdo a Totalmente en Desacuerdo. Altos puntajes indican alto nivel global de autoestima. Cabe aclarar que este instrumento fue validado transculturalmente. 


\section{Evaluación de los rasgos de personalidad.}

Se administró el Big Five Inventory (BFI; Benet-Martinez y John, 1998). Este instrumento ha sido usado y validado con mucho éxito en innumerables culturas (Benet-Martinez y John, 1998) y contiene una escala que mide Agradabilidad la cual puede estar relacionada con la dimensión modelo de Otros tal y como la definieron Bartholomew y Horowitz (1991).

\section{Correlatos socioculturales del apego romántico.}

Obtuvimos las tasas nacionales de fertilidad del Programa de Desarrollo de las Naciones Unidas (2001) correspondientes a 54 de las 56 culturas estudiadas en este proyecto. Otras variables socioculturales de especial interés que se tuvieron en cuenta han sido el Índice de Desarrollo Humano (United Nations Development Program, 2001), Producto Bruto Interno per capita (United Nations Development Program, 2001) y los perfiles nacionales de individualismo vs. colectivismo (Hofstede, 2001).

\section{Resultados}

Consideramos que el vínculo de apego es una característica universal sólo si el $100 \%$ de las regiones poseen el atributo. Por ejemplo, si una escala de apego correlaciona positivamente con una escala de personalidad de la misma manera en todas las culturas, entonces consideramos esta característica como universal cultural. Muchas características han mostrado este nivel de estabilidad en las 62 regiones. Aun así, existen razones por las cuales una cultura puede no mostrar este tipo de relaciones a pesar de que esta relación se de en todas las otras regiones (ver Brown, 1991; Lonner, 1990).

Decimos que un atributo es cercano a lo universal si esta presente en mas del $74 \%$ pero menos del $100 \%$ de las culturas. También utilizamos el termino "tendencia predominante" para describir actitudes mostradas en más del $50 \%$ pero menos del $75 \%$ de las culturas.

\section{1: Determinar si las escalas Modelo de Si Mismo y Modelo de Otros son validas en todas las culturas estudiadas por el ISDP}

Evaluamos la validez de constructo de los modelos de Si Mismo y de Otros 
del RQ (Bartholomew y Horowitz, 1991; Griffin y Bartholomew, 1994 a) determinando si las escalas correlacionaban con un criterio externo de manera consistente en todas las culturas.

\section{Modelo de Sí Mismo}

Primero examinamos si la escala modelo de Si Mismo correlacionaba con una medida de autoestima. En casi todas las regiones culturales ( 55 de 60, esto es, 92 $\%$ ) el modelo de Si Mismo a correlaciona positivamente con autoestima. Ello brinda evidencia transcultural suficiente para considerar como cercana a lo universal la validez convergente del modelo de Si Mismo.

El segundo camino que se ha tomado para evaluar la validez de constructo del modelo de Si Mismo fue correlacionar la escala con alguna medida que estuviese relativamente no asociada con miradas positivas del si mismo. Una de esas escalas es la escala Agradabilidad del Big Five Inventory. El modelo de Si Mismo estadísticamente no correlaciona con Agradabilidad en el $70 \%$ de las culturas del ISDP lo que muestra evidencia transcultural acerca de su validez discriminante. Globalmente, la escala de Si Mismo posee validez de constructo a través de las culturas, apoyando la hipótesis de que el modelo interno de trabajo sobre el Si Mismo es un componente fundamental de los estilos de apego.

\section{Modelo de Otros}

Se evaluó la validez de la escala modelo de Otros examinando si los puntajes correlacionan positivamente con una medida de conducta prosocial. En la mayoría de las regiones culturales (75\%), la escala modelo de Otros correlaciona de forma positiva con Agradabilidad, por lo que se puede considerar como cercana a lo universal su validez convergente.

La segunda vía para evaluar la validez del modelo de Otros fue relacionarla con una medida que no estuviese relacionada con miradas positivas sobre los otros, como por ejemplo, autoestima. La escala modelo de Otros, efectivamente, correlaciona negativamente con autoestima en $65 \%$ de las culturas del ISDP, lo que muestra una tendencia predominante en apoyo de la validez discriminante de la escala modelo de Otros. Globalmente, posee validez de constructo a través de todas las culturas, reafirmando la propuesta general de que el modelo interno de trabajo (working model) recupera vínculos de apego. 


\section{2. ¿Los modelos de Si Mismo y Otros, ¿subyacen a varios de los esti- los de apego romántico de la misma manera en todas las culturas?}

El segundo objetivo de este estudio fue probar la universalidad del modelo de dos dimensiones/cuatro categorías postulado por Bartholomew y Horowitz (1991). De acuerdo con esta visión del apego, ambos modelos deberían ser constructos ortogonales que subyacen de forma independiente a las formas de apego seguro e inseguro de manera sistemática.

\section{Los modelos de Si Mismo y Otros no deberían correlacionar.}

Encontramos evidencia de la universalidad del modelo ortogonal de las dimensiones Si Mismo y Otros. En 50 de las 62 culturas del ISDP (80 \%) la correlación entre modelo de Si Mismo y Otros fue no significativa. En diez regiones culturales, las dimensiones estuvieron correlacionadas positivamente; en dos regiones culturales correlacionan negativamente. En cada caso, sin embargo, las correlaciones significativas fueron pequeñas en su magnitud (cerca de $r=[.16]$ ). Estos hallazgos son similares a los obtenidos por Bartholomew y Horowitz (1991) quienes informan un hallazgo cercano a lo universal en el que los modelos de $\mathrm{Si}$ Mismo y Otros son constructos independientes en la mayoría de las culturas.

\section{El apego romántico seguro debería correlacionar negativamente con el apego temeroso/miedoso}

Si las cuatro escalas del RQ forman una estructura bidimensional con constructos independientes de si mismo y otros, entonces la escala debería correlacionar negativamente con la escala temeroso/miedoso ya que el apego romántico seguro contiene modelos internos de trabajo positivos de Si Mismo y Otros mientras que el apego romántico temeroso/miedoso contiene precisamente lo contrario - modelos internos de trabajo negativos de si mismo y otros. Bartholomew y Horowitz (1991) reportaron originalmente una correlación negativa significativa entre niveles de apego seguro y miedoso: $\mathrm{r}(75)=-0,55, \mathrm{p}<.001$.

En el presente estudio se encontró que en 39 de las 62 culturas $(63 \%)$ el apego seguro correlaciona negativamente con el apego temeroso/miedoso. Aunque casi todas las regiones de Estados Unidos y Canadá mostraron el patrón esperado, la relación negativa entre apego seguro y temeroso/miedoso, solamente calificaron como tendencia prevalerte en todas las regiones culturales del ISDP. Las mayores excepciones a esta regla se dieron en siete muestras de África y en 
muchas de las muestras de regiones asiáticas. En contraste, el patrón esperado se dio claramente, rozando lo cercano a lo universal, en Europa occidental (89\%), Norteamérica (88\%), Europa del sur $(86 \%)$ y entre las culturas más occidentalizadas de Australia y Nueva Zelanda.

Aparentemente, la bipolaridad que se forma entre apego seguro y temeroso/miedoso está limitada a culturas occidentalizadas. Es posible que los problemas de traducción hayan causado la falta de correlación entre muestras no anglo parlantes. Por otro lado, la magnitud de cada correlación, incluso entre muestras de culturas anglo parlantes fue menor a la reportada por Bartholomew y Horowitz (1991). En general, esta prueba proveyó apoyo parcial al modelo de cuatro categorías de apego romántico.

\section{El apego romántico preocupado debería correlacionar negativamen- te con el estilo despreocupado}

Si las cuatro escalas del RQ forman una estructura bidimensional con dimensiones ortogonales independientes de si mismo y otros, entonces la escala preocupado debería correlacionar negativamente con la escala despreocupado. El apego romántico preocupado posee un modelo negativo de si mismo y un modelo positivo de otros mientras que el apego romántico despreocupado posee un modelo positivo de si mismo y un modelo de otros negativo. Barholomew y Horowitz (1991) reportan correlaciones negativas significativas entre auto informes de apegos románticos despreocupados y preocupados: $\mathrm{r}(0,75)=-0,50, \mathrm{p}<0,01$.

Se ha encontrado que sólo el $25 \%$ de las regiones culturales mostraba este patrón. Esto impide calificar la correlación negativa entre apego despreocupado y preocupado como cualquier universal cultural. Como en la relación entre apego seguro y temeroso, la evidencia a favor del modelo de cuatro categorías parece limitada a culturas occidentales, especialmente Estados Unidos y Canadá. Una excepción a la regla es el caso de culturas orientales como India, Taiwán y Hong Kong en las que la evidencia empírica a favor del modelo de cuatro categorías es total.

\section{Cuatro escalas deberían formar dos dimensiones}

Un camino alternativo para evaluar la universalidad del modelo de dos dimensiones/cuatro categorías fue explorar la estructura factorial interna del RQ a través de todas las culturas. Se usaron las técnicas factoriales analíticas utilizadas por Bartholomew y Horowitz (1991). Un análisis de componentes principales usando rotación Varimax de los cuatro ítemes de apego romántico reveló que, 
tanto a nivel de cultura individual como a nivel de región mundial, dos factores eran apropiados para explicar la variación en las escalas de apego romántico. A través de las 10 regiones mundiales, por ejemplo, surgieron dos dimensiones con eigenvalues por encima de 1.0 que explican el 64,8\% de la varianza en Norteamérica, $62 \%$ en Sudamérica, 65,3\% en Europa del Oeste, $61,1 \%$ en Europa del Este, 63,1\% en Europa del Sur, 61,2\% en Medio Oriente, 59,9\% en África, 65,4\% en Oceanía, 58,4 en Sur/sudeste de Asia y el 61,7 de la varianza en Asia del Este. Cabe afirmar que la estructura bidimensional de apego romántico es un universal cultural.

\section{3. ¿Es la forma segura de apego romántico normativa en todas las culturas?}

De acuerdo con la hipótesis normativa (van Ijzendoorn \& Sagi, 1999), la forma segura de apego romántico es una característica de la especie humana, y debería ser la más observada en las diferentes culturas.

Se ha encontrado evidencia que apoya la hipótesis normativa. El estilo de apego romántico obtuvo las evaluaciones más altas en 79\% (49 de 62) de las regiones. Es importante destacar que Sudamérica, y en especial Argentina, es una de las regiones que tiene mayor porcentaje de presencia de apego seguro (ver gráfico 2) en comparación con otras regiones del continente americano (p.ej., Norteamérica).

Grafico 2. Medias del estilo de apego seguro para América del Norte y del Sur

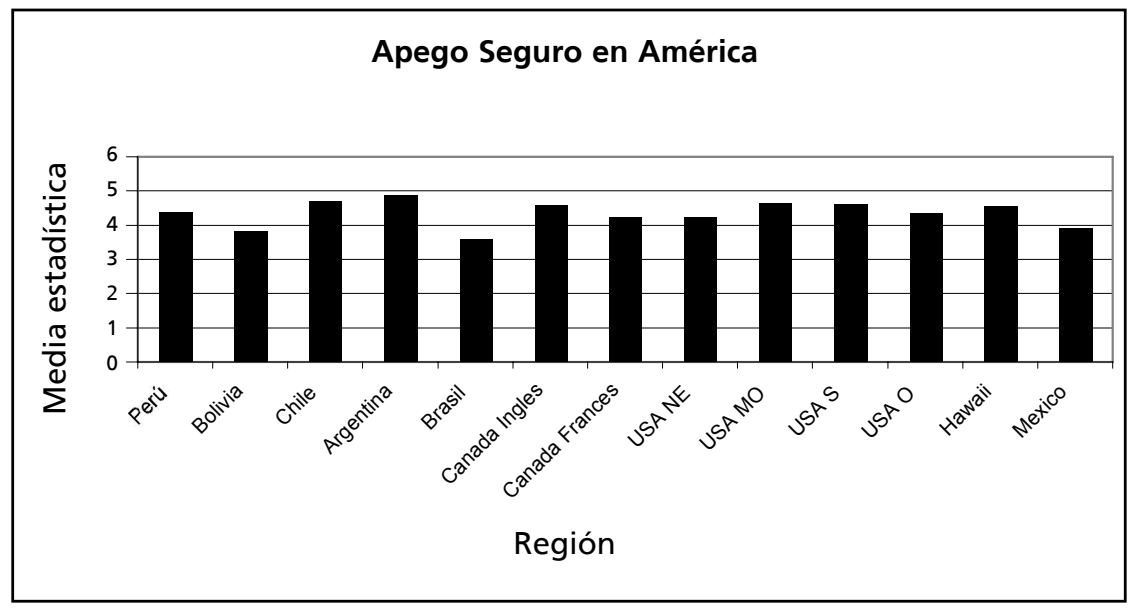


Además, cuando el estilo de apego seguro puntúa más bajo que las otras formas de apego, en muchas de las culturas estas diferencias no son significativas. Algunos hallazgos fueron opuestos a la hipótesis normativa.

\section{4. ¿Los asiáticos del este puntúan particularmente alto en el estilo preocupado de apego romántico?}

Encontramos que los sujetos tanto del sur/sudeste de Asia $(\mathrm{M}=3.95)$ como los del este de Asia $(M=3.98)$ tienden a obtener niveles elevados de apego preocupado en comparación con otros. Esta relación parece apoyar la visión de que en muchas culturas asiáticas la validación (en este caso, validación romántica) es altamente dependiente de la opinión de otros (Markus \& Kitayama, 1991). Esta relación, además, es consistente con la variación cultural en el apego en chicos (van Ijzendoorn \& Sagi, 1999) y con estudios previos comparando los estilos de apego romántico entre asiáticos del este y culturas americanas (Soon \& Malley-Morrison, 2000).

Las naciones que presentan puntuaciones altas en apego romántico preocupado tienden a ser menos individualistas (o más colectivistas) en su orientación que las naciones bajas en apego romántico preocupado. Esta asociación parece funcionar bien en culturas asiáticas. En la figura 1 se muestran los puntajes obtenidos en las escalas modelos de Si Mismo y modelo de Otros en las 10 regiones del ISDP.

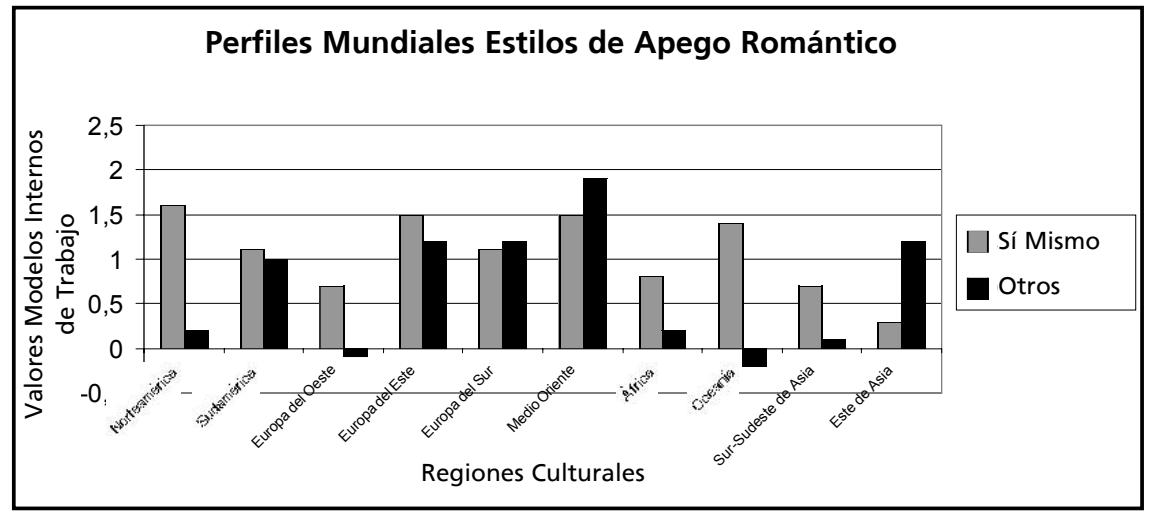

El Este asiático es peculiar en el sentido de que es la única región en la que el modelo de Otro puntúa mas alto que el modelo de Si Mismo. Este hallazgo, mas allá del nivel de universal cultural ya discutido, apoya la hipótesis de que los individuos de naciones en las que la población esta inclinada a juzgar el si mismo en términos de interconexión y el valor que le proveen otros (Markus \& Kitayama, 1999) tienden a esforzarse por lograr el apoyo de otros altamente valorados en el 
contexto especifico de las relaciones románticas.

\section{Poniendo a prueba teorías evolutivas sobre el apego romántico}

De acuerdo con Chisholm (1996, 1999), en culturas con recursos escasos, la estrategia de elección de pareja más eficaz consiste en reproducirse tempranamente y de forma frecuente, y se relaciona con altas tasas de fertilidad y apego inseguro. En culturas con recursos abundantes, la estrategia óptima es invertir fuertemente en pocos hijos y está asociada con tasas de fertilidad bajas y apego romántico seguro. Encontramos evidencia para la teoría de Chisholm relacionando las tasas nacionales de fertilidad con los hallazgos del ISDP sobre apego. Basados en 54 tasas de fertilidad provistas por las Naciones Unidas (United Nations Development Program, 2001) encontramos que las naciones con altas tasas de fertilidad presentan estilos de apego romántico de tipo temeroso/miedoso, $\mathrm{r}(52)=+0,38, \mathrm{p}<0,1$, y de tipo despreocupado, $\mathrm{r}(52)=+0,32, \mathrm{p}<.0,5$.

Tal como lo señalaron Belsky y colaboradores (1991), ciertas experiencias sociales pueden afectar los estilos de apego romántico en el sentido evolutivoadaptativo. Las personas que están expuestas socialmente a mayores niveles de estrés - especialmente paternidades de tipo insensitivas/inconsistentes, ambientes físicamente violentos y graves inconvenientes económicos - tienden a desarrollar estilos de apego inseguros asociados con estrategias de elección de pareja de corto plazo (Schmitt, 2003). Sujetos de contextos sociales con estrés más bajo, como personas que viven en culturas con amplios recursos, tienden a desarrollar estilos de apego romántico seguros asociados con estrategias de elección de pareja monógamas (ver también Rohner \& Britner, 2002; Belsky, 1997, 1999).

Una medida adicional de estrés y gravedad económica puede derivar del índice de Producto Bruto Interno per capita. Se encontró que un PBI per capita alto está asociado con bajos niveles de apego despreocupado, $\mathrm{r}(52)=-0,34, \mathrm{p}<.01$, con bajos niveles de apego preocupado, $\mathrm{r}(52)=-0,43, \mathrm{p}<.001$. Todos estos hallazgos apoyan las premisas de Belsky (1997), Chisholm (1996), y otros autores que sugirieron que contextos más estresantes (p.ej., alta fertilidad, bajo desarrollo humano y pocos recursos) causan un incremento en estilos de apego inseguro, incremento presumiblemente relacionado con estrategias de elección de pareja de corto plazo (Kirkpatrick, 1998; Schmitt y cols, en prensa).

\section{Discusión}

Se ha logrado cumplir con los cinco objetivos primarios a partir de evaluar los estilos de apego romántico adulto en 62 regiones culturales. En primer lugar, 
brindamos evidencia de que las dimensiones Modelo de Si Mismo y del Otro son válidas en la mayor parte de las culturas estudiadas. En segundo término, encontramos que en esas dimensiones Modelo de Si Mismo y Otros no siempre aparecen las cuatro categorías de apego, de la misma manera, en todas las culturas. En tercer lugar, hallamos que la forma segura de apego romántico es normativa en el $79 \%$ de las regiones culturales estudiadas. Confirmamos, en cuarto lugar, que entre los asiáticos del Este predomina el estilo de apego preocupado. Por ultimo, hay evidencia que permite afirmar que los estilos de apego inseguros están asociados con una tasa de fertilidad alta, bajo desarrollo humano y bajos niveles de recursos; asimismo, brinda apoyo empírico adicional a varias teorías evolucionistas del apego romántico. Antes de seguir discutiendo los resultados, es importante señalar las severas limitaciones metodológicas de este estudio.

\section{Limitaciones y futuras direcciones en la investigación}

Una de las precauciones que debe tenerse es que el instrumento utilizado, el Relationship Questionnaire, posee sólo cuatro subescalas, con cada estilo de apego romántico medido sólo por un ítem cada uno. El RQ de cuatro ítemes ha sido utilizado previamente (Bartholomew \& Horowitz, 1991) y es independiente de otras medidas existentes del apego romántico (Leak \& Parsons, 2001). Asímismo, las escalas de un solo ítem pueden complementar instrumentos con escalas multi ítemes (Barrett \& Paltiel, 1996). Sin embargo, creemos que el uso del RQ puede dar pie a futuras investigaciones utilizando instrumentos que midan apego romántico y que posean un formato de escalas multi ítem (Brennan et al, 1998). Las incertidumbres permanecerán hasta desarrollar un instrumento que capte todo el espectro del apego romántico midiendo los mismos constructos que el Adult Attachment Interview (Bartholomew \& Shaver, 1998); también importa conocer si el apego infantil es un precursor del apego romántico adulto (Waters et al, 2000). Los investigadores no deberían sacar conclusiones acerca de la psicología del apego romántico de regiones culturales particulares o naciones basadas solamente en los resultados que se presentan en este trabajo.

Hay tres razones adicionales por las cuales hay que tener precaución a la hora de interpretar y utilizar los resultados de este estudio. Primero, todas las muestras estuvieron sesgadas al no ser seleccionadas al azar y al no basarse en estimaciones probabilísticas poblacionales (ver Mickelson, Kessler \& Shaver, 1997). El hecho de que en las muestras se hayan controlado las variables edad y sexo y otras variables sociodemográficas, ayudó a poder interpretar que el estilo de apego es producto o está relacionado decisivamente con la cultura y no con otras variables demográficas. Futuras investigaciones deberán usar muestras de mayor representatividad e instrumentos estadísticos de mayor complejidad. 
Una segunda razón para ser precavidos es tener en cuenta que todas fueron muestras de estudiantes universitarios. En la medida de lo posible se trató de incluir a miembros de la comunidad, pero los estilos de apego que se muestran en las diferentes culturas están restringidos a poblaciones universitarias de determinada edad.

La tercera razón es que las traducciones no profesionales hechas a más de 30 lenguas diferentes abre la posibilidad de que hayan sido pobres y, en algunos casos se hubiere cambiado el sentido original del ítem. El procedimiento de retro traducción (back translation) intenta balancear, por un lado, el significado de la traducción original y la validez lingüística de los ítemes preservando la integridad del instrumento original y su constructo. El procedimiento utilizado es una aproximación ética y, de algún modo, no es demasiado sensible a los aspectos particulares de cada cultura.

\section{¿Son los modelos de Si Mismo y Otros constructos panculturales?}

Basándonos en una serie de pruebas estadísticas, originalmente usadas por Bartholomew y Horowitz (1991) encontramos datos conflictivos concerniente a la universalidad de las dos dimensiones/cuatro categorías de la estructura del apego romántico. Existe cierta evidencia de que ese modelo no es universal. Por ejemplo, las escalas de apego del RQ no se correlacionan a través de las culturas como lo predice el modelo de dos dimensiones/cuatro categorías. Las formas de apego romántico miedosa y segura están negativamente correlacionadas en el 63 $\%$ de las culturas. Pero los apegos preocupado y despreocupado están negativamente correlacionados en tan sólo el $25 \%$ de las culturas, muestra de la inconsistencia de la universalidad del modelo. De particular importancia es el hecho que ciertas regiones culturales, no justamente aquellas con tamaños de muestra pequeños, son en las que no se ha comprobado la universalidad del modelo. Por ejemplo, ninguna de las siete culturas africanas exhibe una correlación entre apego seguro y miedoso. En el sudeste de Asia, las relaciones entre apego seguro y miedoso son igualmente inconsistentes. Es más, los análisis factoriales demostraron que las cuatro categorías de apego romántico no se encuadran según lo que se suponía en las regiones de Sudamérica, Europa occidental, Europa Oriental, Medio Oriente, África y Asia del Este. Por otro lado, existe una considerable evidencia sobre la universalidad de la estructura bidimensional del apego romántico. Por ejemplo, los resultados de los análisis factoriales sugieren la existencia de dos dimensiones de apego romántico en todas las regiones mundiales. $\mathrm{Y}$ en casi todas las culturas individuales los modelos de si mismo y otro forman dimensiones independientes. Además, existe evidencia respecto a la validez discriminante y convergente para las escalas del modelo de si mismo y otro en la 
mayoría de las culturas individuales. Las personas con un modelo de si mismo más positivo tienen mayor autovaloración. Pero no tienen alto nivel de conductas prosociales. Individuos con modelos positivos del otro tienen alto nivel de conductas prosociales pero no tienen alta autovaloración. Todos estos hallazgos pueden ser considerados " cercanos a lo universal".

A pesar de que es difícil realizar inferencias dadas las limitaciones de muestreo y los hallazgos estadísticos contradictorios, pensamos que es razonable concluir que en casi todas las culturas las personas poseen actitudes cognitivo emocionales básicas que constituyen el modelo de apego romántico sobre la base de las imágenes de si mismo y del otro. Estos modelos internos de trabajo existen como constructos panculturales formando dimensiones independientes que subyacen a los tipos de apego romántico en todas las culturas. Sin embargo, las cuatro categorías de apego romántico de Bartholomew y Horowitz (1991) no parecen encajar en las dos dimensiones a lo largo de todas la regiones del mundo.

\section{Apego romántico seguro: ¿es normativo?}

Según la hipótesis normativa, el apego romántico seguro debe ser el tipo de apego romántico predominante a través de todas las culturas. Dado el amplio numero de culturas presentes en el ISDP, debiéramos obtener evidencia suficiente par comprobar esta hipótesis. Encontramos que el apego seguro es predominante en el $79 \%$ de las culturas estudiadas calificándolo como casi universal. Sin embargo el apego romántico seguro es significativamente más bajo que el despreocupado, preocupado y miedoso en muchas culturas. Estos resultados brindan apoyo empírico limitado a la hipótesis normativa. ¿Por qué el apego romántico no es la forma predominante en todas las culturas? Pueden considerarse tres posibilidades:

1) las ecologías locales de algunas culturas individuales pueden originar formas más inseguras de apego romántico y conductas de elección de pareja. Por ejemplo, en muchas culturas africanas (culturas que experimentan altos niveles de estrés) las formas de apego romántico inseguro tienden a ser altas.

2) Fuerzas socio históricas hacen que ciertas persona exhiban conductas más interdependientes o colectivistas que impactan en sus orientaciones de apego romántico (Markus \& Kitayama, 1991).

3) Las variaciones geográficas en el apego romántico pueden estar causadas por factores religiosos, políticos o económicos compartidos. 


\section{¿Son los asiáticos del este proclives al apego preocupado?}

Encontramos que en las culturas de Asia del Este predomina el estilo de apego preocupado. Este resultado refleja que en estas culturas es altamente importante la opinión de los otros.

\section{Teorías evolucionistas del apego romántico}

Algunos teóricos evolucionistas han hipotetizado que algunos aspectos claves de la cultura están relacionados con el estilo de apego romántico (Belsky et al, 1991). Según estas teorías, las experiencias sociales tempranas llevan a los niños a dos caminos reproductivos: aquellas personas que están expuestas a altos niveles de estrés tienden a desarrollar estilos de apego romántico inseguro y tasas de reproducción mas prolíficas (Cheeseholm, 1996). Individuos de contextos sociales con bajo estrés, desarrollan estilos de apego romántico seguros. Aquellas naciones que poseen altas tasas de fertilidad tienen altos niveles de apego miedoso y despreocupado. Aquellas naciones con bajos índices de desarrollo humano tienen altos niveles de apego preocupado, miedoso y despreocupado. Aquellas culturas con bajo PBI per capita muestran altos niveles de apego preocupado y despreocupado. La relación más consistente se da entre estrés cultural y apego despreocupado, hallazgo consistente con la hipótesis de Kirkpatrick (1998): el apego romántico despreocupado entre las más variadas formas de apego inseguro esta estrechamente relacionado con estrategias de elección de pareja a corto plazo.

\section{Comentarios finales}

Ninguna cultura, en nuestro estudio, revela orientaciones de apego homogéneas y consistentes, es decir, existe una variación intracultural importante que debe ser tomada en cuenta. Algunos autores podrán agrupar las 62 naciones en diferentes regiones culturales. Futuras investigaciones deberán estudiar las relaciones entre geografía, religión, política y estilos de apego.

\section{Referencias Bibliograficas}

Ainsworth, M.D.S., y Marvin, R.S. (1995). On the shaping of attachment theory and research: An interview with Mary D. S. Ainsworth (Fall 1994). Monographs of the Society for Research in Child Development, 60, 3-21. 
Patrones y peculiaridades del apego romántico en adultos de 62 regiones culturales: ¿Son los Modelos de Sí Mismo y Otros constructos panculturales?

Barrett, P.T., y Paltiel, L. (1996). Can a single item replace an entire scale? POP versus the OPQ 5.2. Selection and Development Review, 12, 1-4.

Bartholomew, K. (1990). Avoidance of intimacy: An attachment perspective. Journal of Social and Personal Relationships, 7, 147-178.

Bartholomew, K. (1994). Assessment of individual differences in adult attachment. Psychological Inquiry, 5, 23-27.

Bartholomew, K. y Horowitz, L. M. (1991). Attachment styles in young adults: A test of a four-category model. Journal of Personality and Social Psychology, 62, 226-244.

Bartholomew, K. y Shaver, P.R. (1998). Methods of assessing adult attachment: Do they converge? In J.A. Simpson \& W.S. Rholes (Eds.), Attachment theory and close relationships (pp. 25-45). New York: Guilford.

Belsky, J. (1999). Modern evolutionary theory and patterns of attachment.. In J. Cassidy y P.R. Shaver (Eds.), Handbook of attachment (pp. 141-161). New York: Guilford.

Belsky, J., Steinberg, L., y Draper, P. (1991). Childhood experience, interpersonal development, and reproductive strategy: An evolutionary theory of socialization. Child Development, 62, 647-670.

Benet-Martinez, V., y John, O.P. (1998). Los Cinco Grandes across cultures and ethnic groups: Multitrait-multimethod analyses of the Big Five in Spanish and English. Journal of Personality and Social Psychology, 75, 729-750.

Bowlby, J. (1969/1982). Attachment and loss: Vol. I. Attachment. New York: Basic Books.

Bowlby, J. (1973). Attachment and loss: Vol. II. Separation: Anxiety and anger. New York: Basic Books.

Bowlby, J. (1980). Attachment and loss: Vol. III. Loss. New York: Basic Books.

Bowlby, J. (1988). A secure base: Parent-child attachment and healthy human development. New York: Basic Books.

Brennan, K.A., Clark, C.L., y Shaver, P.R. (1998). Self-report measurement of adult attachment: An integrative overview. In J.A. Simpson y W.S. Rholes (Eds.), Attachment theory and close relationships (pp. 46-76). New York: Guilford. 
Brennan, K.A., y Shaver, P.R. (1995). Dimensions of adult attachment, affect regulation, and romantic relationship functioning. Personality and Social Psychology Bulletin, 21, 267-283.

Bretherton, I., y Munholland, K.A. (1999). Internal working models in attachment relationships: A construct revisited. In J. Cassidy y P.R. Shaver (Eds.), Handbook of attachment (pp. 89-111). New York: Guilford.

Brown, D.E. (1991). Human universals. New York: McGraw-Hill, Inc.

Carver, C.S. (1997). Adult attachment and personality: Converging evidence and a new measure. Personality and Social Psychology Bulletin, 23, 865-883.

Cassidy, J., y Shaver, P. (1999) (Eds.). Handbook of attachment: Theory, research, and clinical applications. New York: Guilford Press.

Chisholm, J.S. (1996). The evolutionary ecology of attachment organization. Human Nature, 7, 1-38.

Chisholm, J.S. (1999). Steps to an evolutionary ecology of the mind. In A.L. Hinton (Ed.), Biocultural approaches to the emotions (pp. 117-149). Cambridge, United Kingdom: Cambridge University Press.

Collins, N., y Read, S. (1990). Adult attachment relationships, working models and relationship quality in dating couples. Journal of Personality and Social Psychology, 58, 644-683.

Feeney, J.A. (1999). Adult romantic attachment and couple relationships. In J. Cassidy y P.R. Shaver (Eds.), Handbook of attachment (pp. 355-377). New York: Guilford.

Griffin, D., y Bartholomew, K. (1994a). Models of self and other: Fundamental dimensions underlying measures of adult attachment. Journal of Personality and Social Psychology, 67, 430-445.

Griffin, D., y Bartholomew, K. (1994b). The meta-physics of measurement: The case of adult attachment. In K. Bartholomew and D. Perlman (Eds.), Attachment processes in Advances in personal relationships (Vol. 5, pp. 17-52). London: Jessica Kingsley.

Hazan, C., y Shaver, P.R. (1987). Romantic love conceptualized as an attachment process. Journal of Personality and Social Psychology, 52, 511-524. 
Hazan, C., y Zeifman, D. (1999). Pair bonds as attachments: Evaluating the evidence. In J. Cassidy y P.R. Shaver (Eds.), Handbook of attachment (pp. 336-354). New York: Guilford.

Kirkpatrick, L.A. (1998). Evolution, pair-bonding, and reproductive strategies: A reconceptualization of adult attachment.. In J.A. Simpson y W.S. Rholes (Eds.), Attachment theory and close relationships (pp. 353-393). New York: Guilford.

Kirkpatrick, L.A., y Hazan, C. (1994). Attachment styles and close relationships: A four year prospective study. Personal Relationships, 1, 123-142.

Leak, G.K., y Parsons, C.J. (2001). The susceptibility of three attachment style measures to socially desirable responding. Social Behavior and Personality, 29, 21-30.

Lonner, W.J. (1980). The search for psychological universals. In H.C. Triandis y W.W. Lambert (Eds.), Handbook of cross-cultural psychology (Vol. 1, pp. 143204). Boston, MA: Allyn y Bacon.

Main, M. (1990). Cross-cultural strategies of attachment and attachment organization: Recent studies, changing methodologies, and the concept of conditional strategies. Human Development, 33, 48-61.

Markus, H.R., y Kitayama, S. (1991). Culture and the self: Implications for cognition, emotion, and motivation. Psychological Review, 98, 224-253.

Marvin, R.A., y Britner, P.A. (1999). Normative development: The ontogeny of attachment. In J. Cassidy y P.R. Shaver (Eds.), Handbook of attachment (pp. 4467). New York: Guilford.

Mickelson, K.D., Kessler, R.C., y Shaver, P.R. (1997). Adult attachment in a nationally representative sample. Journal of Personality and Social Psychology, 73, 1092-1106.

Rholes, W.S., Simpson, J.A., y Stevens, J.G. (1998). Attachment orientations, social support, and conflict resolution in close relationships. In J.A. Simpson y W.S. Rholes (Eds.), Attachment theory and close relationships (pp. 166-188). New York: Guilford.

Roberts, N., y Noller, P. (1998). The associations between adult attachment and couple violence: The role of communication patterns and relationship satisfaction. In J.A. Simpson y W.S. Rholes (Eds.), Attachment theory and close relationships (pp. 317-350). New York: Guilford. 
Rohner, R.P., y Britner, P.A. (2002). Worldwide mental health correlates of parental acceptance-rejection: Review of cross-cultural and intracultural evidence. Cross-Cultural Research, 36, 16-47.

Rosenberg, M. (1965). Society and the adolescent self-image. Princeton, NJ: Princeton University Press.

Rothbaum, F., Weisz, J., Pott, M., Miyake, K., y Morelli, G. (2000). Attachment and culture: Security in the United States and Japan. American Psychologist, 55, 1093-1104.

Scharfe, E., y Bartholomew, K. (1994). Reliability and stability of adult attachment patterns. Personal Relationships, 1, 23-43.

Schmitt, D.P. (2003). On the psychological origins of sexual promiscuity: A cross-cultural study of personality, romantic attachment, and sociosexuality. Manuscript submitted for publication

Schmitt, D.P., Alcalay, L., Allensworth, M., Allik, J., Ault, L., Austers, I. et al. (in press). Are men universally more dismissing than women? Gender differences in romantic attachment across 62 cultural regions. Personal Relationships.

Simpson, J.A. (1990). Influences of attachment styles on romantic relationships. Journal of Personality and Social Psychology, 59, 971-980.

Simpson, J.A. (1999). Attachment theory in modern evolutionary perspective. In J. Cassidy y P.R. Shaver (Eds.), Handbook of attachment (pp. 115-140). New York: Guilford.

Simpson, J.A., y Rholes, W.S. (1998) (Eds.). Attachment theory and close relationships. New York: Guilford Press.

Soon, H., y Malley-Morrison, K. (2000). Young adult attachment styles and intimate relationships with close friends: A cross-cultural study of Koreans and Caucasian Americans. Journal of Cross-Cultural Psychology, 31, 528-534.

Sümer, N., y Güngör, D. (1999). Psychometric evaluation of adult attachment measures in Turkish samples and a cross-cultural comparison. Turkish Journal of Psychology, 14, 71-109.

Suomi, S.J. (1995). Influence of Bowlby's attachment theory on research on nonhuman primate biobehavioral development. In S. Goldberg, R. Muir, y J. Kerr 
Patrones y peculiaridades del apego romántico en adultos de 62 regiones culturales: ¿Son los Modelos de Sí Mismo y Otros constructos panculturales?

(Eds.), Attachment theory: Social, developmental, and clinical perspectives (pp. 185-201). Hillsdale, NJ: Analytic Press.

United Nations Development Programme (2001). Human development report 2001. New York: Oxford University Press.

van IJzendoorn, M.H., y Sagi, A. (1999). Cross-cultural patterns of attachment: Universal and contextual dimensions. In J. Cassidy y P.R. Shaver (Eds.), Handbook of attachment (pp. 713-734). New York: Guilford.

Waters, E., Merrick, S., Treboux, D., Crowell, J., y Alberstein, L. (2000). Attachment security in infancy and early adulthood: A twenty-year longitudinal study. Child Development, 71, 684-689. 